\title{
VARIATION IN THE YAK CALPASTATIN GENE (CAST)
}

\author{
YANG G. ${ }^{1}$, ZHOU H. ${ }^{2}$, HU J.1, LUO Y. ${ }^{*}$ AND HICKFORD J.G.H. ${ }^{2 *}$ \\ ${ }^{1}$ Gansu Key Laboratory of Herbivorous Animal Biotechnology, Gansu Agricultural University, Lanzhou, China. \\ ${ }^{2}$ Faculty of Agriculture and Life Sciences, Lincoln University, Lincoln, New Zealand. \\ *Corresponding Author: Email-yzluo@public.lz.gs.cn, Jonathan.Hickford@lincoln.ac.nz
}

Received: February 14, 2012; Accepted: March 09, 2012

\begin{abstract}
Calpastatin (CAST) is a specific inhibitor of calpain (calcium-dependent cysteine protease). This study investigated the potential for variation in yak (Bos grennies) CAST. PCR-SSCP analysis of exon 6 of yak CAST revealed three unique patterns (named A-C). Sequencing of the amplicons revealed two nucleotide substitutions. One substitution (c.398G/C) would nominally change the amino acid sequence (p.S133T) of yak calpastatin. The variant sequence $A$ which carried c.398C was the most common in the yaks tested (95.1\%). This is the first report that found yak CAST is variable, and as in pigs, sheep and cattle, this variation may affect animal production traits.
\end{abstract}

Key words- CAST, genetic variation, Yak (Bos grennies), PCR- single strand conformational polymorphism (PCR-SSCP).

Citation: Yang G., et al (2012) Variation in the yak calpastatin gene (CAST). International Journal of Genetics, ISSN: 0975-2862 \& E-ISSN: 0975-9158, Volume 4, Issue 1, pp.-72-73.

Copyright: Copyright@2012 Yang G., et al. This is an open-access article distributed under the terms of the Creative Commons Attribution License, which permits unrestricted use, distribution, and reproduction in any medium, provided the original author and source are credited.

\section{Introduction}

Calpastatin (CAST) is a specific inhibitor of calpain (the calciumdependent cysteine protease). It affects meat tenderisation by binding with calpain, which in turn inactivates proteolysis. An increased level of calpastatin in lamb meat was found to be associated with decreased calpain activity, decreased proteolysis and increased toughness [1].

Calpastatin is composed of an $L$ domain and four repetitive calpain inhibitary domains (domain 1-4) [2]. In cattle (Bos taurus), CAST is a multi-promoter gene, and encodes a series of calpastatin isoforms using different alternative splicing processes [3]. Variation in CAST has been revealed for cattle, sheep and pigs [4 -6] and associations between variation in CAST and meat tenderness have been found in pig and cattle [7, 8] and with birth weight and growth until weaning in sheep [9]. Genetic testing based on detecting variation in CAST has been commercialized as a genemarker for improving meat tenderness in cattle [2].

Although a cDNA sequence for yak CAST has been identified [10], variation in the yak gene has not been described previously. In this study, the potential for variation in exon 6 of yak CAST which encodes the L-domain of calpastatin was investigated.

\begin{abstract}
Materials and Methods
DNA sample collection

Blood samples from 216 yaks from nine different farms in three different regions of the Peoples Republic of China (Qinghai Province, Gansu-Tianzhu Region and Gansu-Tibet Region) were collected onto FTA cards (Whatman, Middlesex, UK). A rapid twostep DNA extraction method [11] was used to get purified genomic DNA from these samples.
\end{abstract}

\section{PCR amplification and SSCP analysis}

The yak CAST exon 6 was amplified using PCR primers (5'gttatgaattgctttctactc-3' and 5'-atacgattgagagacttcac-3') as described previously [6]. Amplicons were subject to SSCP analysis using conditions described in Zhou et al. [6].

\section{Sequencing and sequence analysis}

PCR amplicons representative of different SSCP patterns from yaks that were homozygous for CAST were sequenced at the Lincoln University DNA Sequencing Facility.

Sequence alignments, translations and comparisons were carried out using DNAMAN (version 5.2.10, Canada). 


\section{Results}

Three unique SSCP banding patterns were detected for the amplicon from the yak CAST exon 6 region (Fig.1). Either one or two of these patterns were observed in individual yaks, which is consistent with them having homozygous or heterozygous genotypes, respectively. Sequencing confirmed that each pattern represented a novel CAST sequence variant. These sequences were named as $A-C$ and deposited onto GenBank with accession nos. HM466969-HM466971, respectivley.

Two nucleotide substitutions (c.398G/C and c.468C/T) were detected in the exon 6 of CAST (Table 1). The substation c.398G/C would potentially change the amino acid sequence of yak CAST (p.S133T).

Allele frequencies of CAST were determined for 216 yaks from nine different farms in three different regions of the Peoples Republic of China (Qinghai Province, Gansu-Tianzhu Region and the Gansu-Tibet Province). Genotypes $A A(93.5 \%), A B(2.8 \%), A C$ $(0.4 \%), B B(1.4 \%)$ and $C C(1.9 \%)$ were observed, with individual variant frequencies of $95.1 \%(A), 2.8 \%(B)$ and $2.1 \%(C)$ (Table 2$)$.

Table 1- Nucleotide substitutions detected in exon 6 of yak CAST

\begin{tabular}{llll|} 
Substitution $^{1}$ & Variant A & Variant B & Variant C \\
c. $398 \mathrm{C} / \mathrm{G}^{2}$ & $\mathrm{C}$ & $\mathrm{G}$ & $\mathrm{G}$ \\
$\mathrm{c} .468 \mathrm{C} / \mathrm{T}$ & $\mathrm{C}$ & $\mathrm{T}$ & $\mathrm{C}$ \\
\hline
\end{tabular}

${ }^{1}$ The positions of the nucleotide substitutions refer to sequence EU009141.

2 This nucleotide substitution would result in an amino acid change of p.S133T

Table 2- Frequencies of the genotypes and variants of yak CAST

\begin{tabular}{|ll|}
\hline Genotype/Allele & Frequency $(\%)(n=216)$ \\
\hline AA & 93.5 \\
AB & 2.8 \\
AC & 0.4 \\
BB & 1.4 \\
BC & 0 \\
CC & 1.9 \\
A & 95.1 \\
B & 2.8 \\
C & 2.1 \\
\hline
\end{tabular}

\section{Discussion}

Exon 6 encodes the L-domain of the calpastatin which is known to interact with the inactive form of calpain. It plays an important role in the calpain/calpastatin system [12]. Given the crucial role that exon 6 may play in the calpastatin/calpain system, it is therefore seems likely that the variation revealed here in exon 6 of yak CAST could have both structural and functional effects on the calpastatin system and potentially affect the tenderisation of yak meat.

In the 216 yaks investigated, variant $A$ (frequency $95.1 \%$; Table 2) encodes a putative threanine at residue position 133 as opposed to the serine encoded in alleles $B$ and $C$. This nucleotide and amino acid variation has not been recorded to date in sheep or cattle (Fig.2).

Yaks are slow growing due to the extreme environment in which they are found [13]. Given the association found between variation in the CAST and birth weight and growth rate (to weaning) in sheep [9], it is also possible that the low growth performance of yak could be partially explained by having unique variation in CAST. In cattle, Žgur et al. [14] have associated low growth rates with increased tenderness which is, in consistent with the more tender meat and low growth rate found in yak although as discussed by Žgur et al. [14] the influence of growth rate on meat tenderness seems to depend mainly on changes in muscle protein turnover. How this manifests in yak is also yet to be understood, but the variation reported here in CAST suggests are possible deserve for further investigation.

This is the first report of polymorphic variation in yak CAST and we accept that it is limited by the lack of phenotype data from yaks for meat tenderness or growth rate. Future work is now required to investigate more samples and for which these phenotypic data are available.

\section{Acknowledgements}

The financial support from Gene-Marker Laboratory, Lincoln University, New Zealand, is gratefully acknowledged. The authors thank S. O. Byun, S. Kunhareang, Q. Fang and H. Gong for technical assistance.

\section{References}

[1] Geesink G.H. and Koohmaraie M. (1999) Journal of Animal Science, 77, 1490-1501.

[2] Kemp C.M., Sensky P.L., Bardsley, R.G., Buttery P.J. and Parr T. (2010) Meat Science, 84, 248-256.

[3] Raynaud P., Gillard M., Parr T., Bardsley R., Amarger V. and Leveziel H. (2005) Archives of Biochemistry and Biophysics, 440, 46-53.

[4] Zambonelli P., Davoli R., Fontanesi L., Costosi E. and Russo V.A. (1995) Animal Genetics, 26, 373.

[5] Bishop M.D., Koohmaraie M., Killefer J. and Kappes S. (1993) Journal of Animal Science, 71, 2277.

[6] Zhou H., Hickford J.G. and Gong H. (2007) Molecular and Cellular Probes, 21, 242-244.

[7] Casas E., White S.N., Wheeler T.L., Shackelford S. D., Koohmaraie M., Riley D.G., Chase Jr C. C., Johnson D.D. and Smith T.P.L. (2006) Journal of Animal Science, 84, 520-525.

[8] Lindholm-Perry A.K., Rohrer G.A., Holl J.W., Shackelford S.D., Wheeler T.L., Koohmaraie M. and Nonneman D. (2009) Animal Genetics, 40, 713-721.

[9] Byun S.O., Zhou H., Forrest R.H., Frampton C.M. and Hickford J.G. (2008) Animal Genetics, 39, 572-573.

[10]Zhang L., Ma B., Wu J., Fei C., Yang L. and Wan H. (2010) Acta Biochimica Polonica, 57, 35-41.

[11]Zhou H., Hickford J.G., Fang Q. (2006) Analytical Biochemistry, 354, 159-161.

[12]Melloni E., Averna M., Stifanese R., De Tullio R., Defranchi E., Salamino F. and Pontremoli S. (2006) Journal of Biological Chemistry, 281, 24945-24954.

[13]Luo X., Xu J., Li Q., Wei Y. and Zhao X. (2005) Archiv Tierzucht, 48, 555-561.

[14]Žgur S., Čepon M., Čepin S. (2003) Czech Journal of Animal Science, 48, 113-119. 